\title{
Salvage Photodynamic Therapy Using Talaporfin Sodium for Local Failure of Esophageal Squamous Cell Carcinoma
}

\author{
Yuichiro Ikebuchi, ${ }^{\dagger} \dagger$ Akira Yoshida, ${ }^{*} \dagger$ Hiroki Kurumi, $\uparrow$ Yu Kamitani, $\uparrow$ Sho Yasui, $\uparrow$ Yusuke Nakada, $\dagger$ \\ Koichiro Kawaguchi, $\uparrow$ Kazuo Yashima $\uparrow$ and Hajime Isomoto $\dagger$ \\ †Division of Gastroenterology and Nephrology, Department of Multidisciplinary Internal Medicine, School of Medicine, Faculty of \\ Medicine, Tottori University, Yonago 683-8503, Japan
}

\begin{abstract}
Background Talaporfin sodium photodynamic therapy (TS-PDT) for local failure after chemoradiotherapy (CRT) in patients with esophageal squamous cell carcinoma has recently been reported to be highly effective and less invasive, compared to other treatment modalities. TS-PDT was recently introduced at the Tottori University Hospital, Japan. The aim of this study is to clarify the efficacy and safety of PDT in our hospital.
\end{abstract}

Methods This was a single-center observational study. We examined eight cases of TS-PDT performed between January 2016 and December 2019. The main endpoints were local complete remission (L-CR) rate and the adverse events. In addition, age, gender, histology, tumor location, TNM stage, tumor depth, irradiation dose, and overall survival (OS) were examined.

Results The patients included 7 men and a woman, with an average age of 72.1 years (range 63-82 years). The baseline clinical stages before CRT or radiotherapy were stage I in 1 , stage II in 3, stage III in 3, and stage IVA in 1 patient. The $\mathrm{T}$ stage on endoscopic assessment before TS-PDT was T1 in 6 patients and T2 in 2 patients. Treatment outcomes and adverse events were evaluated. There were no treatment-related deaths, and no significant adverse events occurred intraoperatively or postoperatively. The L-CR rate was $7 / 8(87.5 \%)$; T1 cases had $100 \%(6 / 6) \mathrm{L}-\mathrm{CR}$, while T2 cases had 50\% $(1 / 2)$. The 2-year OS rates were $87 \%$.

Conclusion TS-PDT was observed to be safe and effective in the first eight cases of its application following its introduction in our hospital.

Key words esophageal cancer; talaporfin sodium; photodynamic therapy; salvage treatment; squamous cell carcinoma

Esophageal cancer was the sixth most common malignancy worldwide in 2018. ${ }^{1}$ Esophageal adenocarcinoma and esophageal squamous cell carcinoma (ESCC) are the two major histological subtypes of esophageal cancer. In Japan, ESCC is currently the most common subtype. ${ }^{2}$ Chemoradiotherapy (CRT) is a curative treatment option for ESCC. ${ }^{3,4}$ However, local recurrence or residual disease without distant metastasis, after completion of CRT, remains a major problem. ${ }^{5,6}$ Salvage esophagectomy in these patients is associated with higher postoperative morbidity and mortality. 7,8 Salvage endoscopic resection (ER) is a curative treatment option for early ESCC with no lymph node metastasis. However, in case of recurrent lesions after CRT, en bloc and complete resection rates of ER are relatively low due to severe fibrosis. ${ }^{9,} 10$

Photodynamic therapy (PDT), which induces tissue destruction by combining a photosensitizer and laser irradiation, has been developed as a less invasive treatment of choice for a variety of cancers including esophageal cancer. ${ }^{11,12}$ There have been few previous reports studying the efficacy and safety of salvage PDT for local failure in ESCC after CRT. ${ }^{13}, 14$ Yano et al. reported that $76 \%(19 / 25)$ of patients achieved a local complete remission (L-CR) in a phase II study of salvage PDT using a combination of the first generation photosensitizer, porfimer sodium (PS), and excimer dye laser (PS-PDT) for local residual stage T1 ESCC after CRT. ${ }^{13}$ Yano et al. have also reported a high L-CR rate (88.8\%) for local residual stage T1 and T2 tumors in an investigator-initiated trial of PDT using a combination of the second-generation photosensitizer, talaporfin sodium (TS), and diode laser (TS-PDT). ${ }^{14}$ Salvage PDT after local failure of CRT or radiotherapy (RT) was covered by the Japanese health insurance from 2015. We used TS-PDT in January 2016 for the first time in Chugoku District. Herein, we report our experience

*Y.I. and A.Y. equally contributed to this work.

Corresponding author: Yuichiro Ikebuchi, $\mathrm{MD}, \mathrm{PhD}$

ikebu@tottori-u.ac.jp

Received 2020 December 8

Accepted 2021 February 5

Online published 2021 February 22

Abbreviations: CT, Computed tomography; CTCAE, Common Terminology Criteria for Adverse Events; CRT, chemoradiotherapy; ER, endoscopic resection; ESCC, esophageal squamous cell carcinoma; EUS, endoscopic ultrasound; L-CR, local complete response; PDT, Photodynamic therapy; PS, porfimer sodium; RT, radiotherapy; TS, talaporfin sodium; TS-PDT, Talaporfin sodium photodynamic therapy 
Table 1. Characteristics of the patients who underwent PDT at the Tottori University Hospital between January 2016 and December 2019

\begin{tabular}{ccccccc}
\hline Case No & Age & Gender & Initial Treatment & Histology & Location & TNM \\
\hline 1 & 68 & M & CRT & SCC & Mt & II \\
2 & 63 & M & CRT & SCC & Mt & III \\
3 & 74 & M & CRT & SCC & Lt & II \\
4 & 80 & F & CRT & SCC & Mt, Lt & III \\
5 & 73 & M & CRT & SCC & Lt & IVA \\
6 & 69 & M & CT, RT & SCC & Mt & II \\
7 & 68 & M & CRT & SCC & Lt & I \\
8 & 82 & M & CRT & SCC & Ut & III \\
\hline
\end{tabular}

CT, Chemotherapy; CRT, chemoradiotherapy; F, female; Lt, lower thoracic esophagus; M, male; Mt, middle thoracic esophagus; PDT, photodynamic therapy; RT, radiotherapy; SCC, squamous cell carcinoma; TNM, TNM stage (UICC 8th) before PDT; Ut, upper thoracic esophagus.

regarding its safety profile and efficacy, through a case series.

\section{MATERIALS AND METHODS}

This was a single-center observational study. We examined eight cases of PDT performed between January 2016 and December 2019. The indications for salvage PDT included all these criteria: (i) lesions limited to no higher than stage T2 prior to PDT, (ii) local failure after CRT or radiotherapy, (iii) absence of any lymph node or distant metastasis, (iv) no indications for ER, and (v) no indications for, or the patient's refusal to undergo salvage surgery. The aims of this study are to clarify the $\mathrm{CR}$ rate at the primary site and the safety of PDT (adverse events and toxicity). The adverse events and toxicity were evaluated and graded according to the Common Terminology Criteria for Adverse Events (CTCAE) version 4.0. ${ }^{15}$ In addition, age, gender, treatment before PDT, histology, tumor location, TNM stage before PDT, pattern of failure, number of lesions, tumor depth, and irradiation dose were evaluated. The TNM classification of the International Union Against Cancer, 8th edition, was used. The depth of the lesion was evaluated by endoscopic ultrasound (EUS), and in cases where performing EUS was difficult, we used endoscopic white light images for evaluation.

This study was approved by the ethical review board of the Tottori University Hospital (approval number 1508A024).

\section{PDT treatment and surveillance}

All PDTs were performed as inpatient procedures. Every case was performed in an endoscopic room under intravenous sedation. PDT was applied by intravenous administration of $40 \mathrm{mg} / \mathrm{kg}$ TS followed by diode laser irradiation at $100 \mathrm{~J} / \mathrm{cm}^{2}$, six hours later. On the next day, if the treatment efficacy, assessed by ischemic changes, was observed to be insufficient on endoscopy, additional laser irradiation was performed.

After photosensitizer administration, all patients avoided direct sun exposure and stayed in a room maintained at less than 500 Lux. The duration of hospital stay after PDT was set at two weeks, provided there were no complications related to PDT. The endoscopic examination was repeated regularly after $1-2$ weeks to evaluate the response until L-CR was confirmed. Computed tomography (CT) was used to evaluate distant organ or lymph node metastasis every six months thereafter.

\section{RESULTS \\ Patients characteristics}

Between January 2016 and December 2019, a total of 8 patients (10 lesions) with ESCC who had local failure after CRT $(n=7)$ or radiotherapy $(\mathrm{RT})(n=1)$ were studied. The patients included seven men and one woman, with ages ranging $63-82$ years. The CRT regimen was standardized and comprised cisplatin (70 $\mathrm{mg} / \mathrm{m}^{2} /$ day 1$)$ and 5-fluorouracil (700 $\mathrm{mg} / \mathrm{m}^{2} /$ day 1 to 4$)$ with concomitant RT of 54-60 Gy. The tumor location was the upper esophagus in one, middle esophagus in four, and lower esophagus in four patients. The baseline clinical stages before CRT or RT were stage I in one, stage II in three, stage III in three, and stage IVA in one patient (Table 1).

\section{Efficacy and safety}

The details of the procedure and treatment efficacy of TS-PDT are summarized in Table 2. There were four cases of recurrence after achieving CR with CRT/ 
Table 2. The details of the procedure and efficacy of PDT

\begin{tabular}{llcllcll}
\hline Case & $\begin{array}{l}\text { Pattern of } \\
\text { failure }\end{array}$ & $\begin{array}{l}\text { Number of } \\
\text { lesions }\end{array}$ & Tumor depth & $\begin{array}{l}\text { Lesion circumference } \\
\text { of the lumen }\end{array}$ & $\begin{array}{l}\text { Total irradiation } \\
\text { dose }(\mathrm{J})\end{array}$ & $\begin{array}{l}\text { Response } \\
\text { of PDT }\end{array}$ & Adverse event \\
\hline 1 & Recurrence & 1 & Tlb & $1 / 4-1 / 2$ & 500 & L-CR & Fever, pain, stricture \\
\hline 2 & Recurrence & 1 & Tlb & $1 / 4-1 / 2$ & 700 & L-CR & Pain, stricture \\
\hline 3 & Recurrence & 1 & Tlb & $<1 / 4$ & 350 & L-CR & - \\
\hline 4 & Residual & 3 & Tla, Tlb, T1b & $1 / 4-1 / 2$ & 800 & L-CR & Fever \\
\hline 5 & Recurrence & 1 & T1a & $1 / 4-1 / 2$ & 700 & L-CR & Fever \\
\hline 6 & Residual & 1 & Tlb & $<1 / 4$ & 300 & L-CR & Pain \\
\hline 7 & Residual & 1 & T2 & $1 / 4-1 / 2$ & 800 & L-CR & - \\
\hline 8 & Residual & 1 & T2 & $1 / 4-1 / 2$ & 500 & non-CR & - \\
\hline
\end{tabular}

L-CR, local complete response; PDT, photodynamic therapy.

(a)

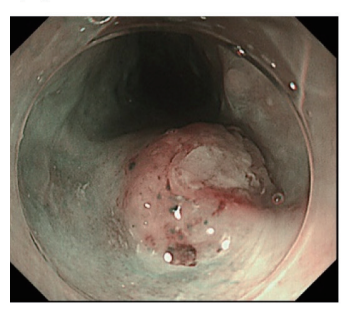

(b)

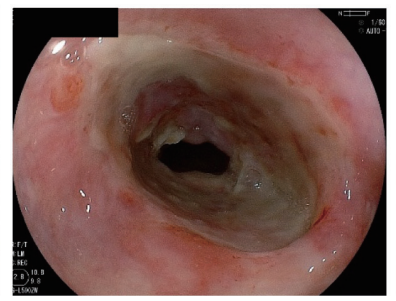

(c)

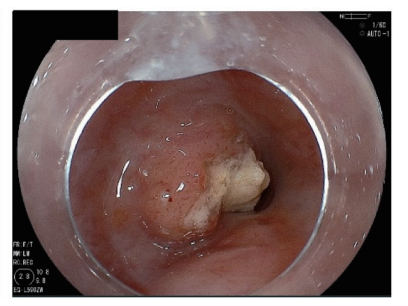

Fig. 1. Endoscopic images of the patient with non-CR after PDT. (a) Residual lesion detected after chemoradiotherapy. (b) Two days after PDT, ischemic changes were observed at the laser-irradiated site. (c) Persistence of lesion two months after PDT. CR, complete remission; L-CR, Local complete remission; PDT, photodynamic therapy.

RT, and four residual lesions following CRT in four patients. Seven patients had one lesion and one patient had two lesions. The T stage on endoscopic assessment before TS-PDT was T1 in 6 patients $(2$ Tla lesion, and $6 \mathrm{~T} 1 \mathrm{~b}$ lesion) and T2 in 2 patients (only one patient had a total of three lesions: one Tla and two T1b lesions). The two lesions of Tla had fibrosis in the submucosa after endoscopic treatment or radiation therapy, and endoscopic treatment was judged to be difficult. None of the patients had lymph node or distant metastasis on enhanced systemic CT evaluation before TS-PDT. The total irradiation dose was 600J (range, 300-800J). Seven patients achieved L-CR and the L-CR rate was $87.5 \%$ (7/8). In the patient with no CR, an ulcer was observed at the laser-irradiated site two days after TS-PDT; however, the residual tumor after CRT did not disappear and persisted even two months after TS-PDT (Fig. 1). A representative case of a patient who achieved $\mathrm{CR}$ is shown in Fig. 2. This case had no recurrence after three years of follow-up (Fig. 2).

There were three adverse events, including stenosis, pain, and fever after TS-PDT. The fever and pain were grade 1 in all the cases according to CTCAE, and most of them recovered within a day. Two patients underwent endoscopic balloon dilation due to esophageal stenosis, one of whom needed an intravenous drip on admission due to the stenosis (CTCAE grade 3). Perforation and skin phototoxicity were not observed in this study. There were no treatment-related deaths.

The median follow-up period was 30 months (range, 14-47 months). The seven patients who achieved L-CR are on follow-up at the outpatient clinic till date. The patient who did not achieve CR (non-CR) had difficulty ingesting orally due to tumor growth 3 months after TS-PDT. After that, the patient continued chemotherapy, however died of disease progression 14 months after TS-PDT. The median overall survival (OS) was unpredictable in all patients. The 2-year OS was $87 \%$ (Fig. 3).

\section{DISCUSSION}

This study examined the results of the first eight cases of TS-PDT in our institution after its introduction. This was also the first application of TS-PDT in the Chugoku district of Japan. There were no treatment-related deaths in our study, and TS-PDT was observed to be effective as a salvage treatment option for local failure after CRT for ESCC, in line with the few prior reports. 
(a)

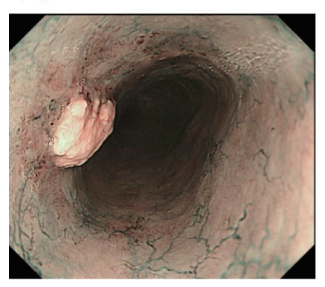

(d) (b)

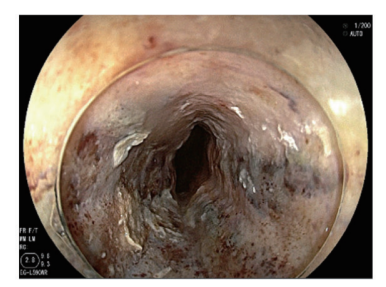

(c)

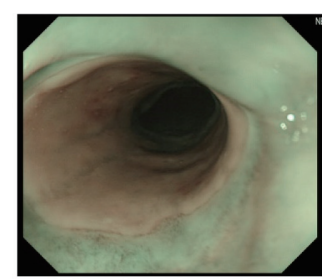

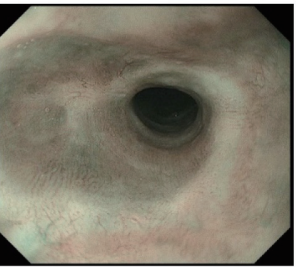

(e)

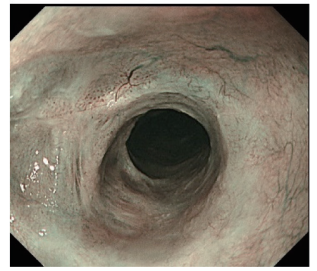

Fig. 2. Representative case of L-CR after PDT, endoscopic images. (a) Residual lesion detected after chemoradiotherapy. (b) Two days after PDT, ischemic changes observed at the laser-irradiated site. (c) Four weeks after PDT, deep ulceration was observed at the site. (d) CR was achieved 10 weeks after PDT. (e) CR maintained three years after PDT. CR, complete remission; L-CR, Local complete response; PDT, photodynamic therapy.

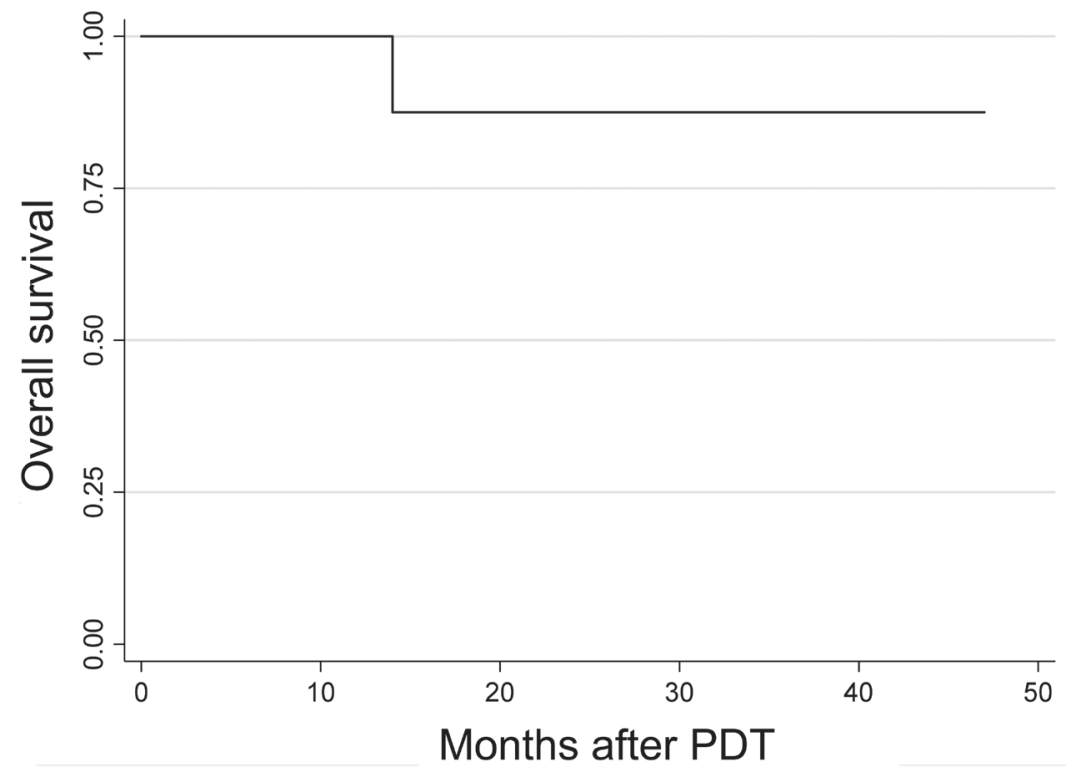

Fig. 3. Survival curves for the enrolled patients after photodynamic therapy. PDT, photodynamic therapy.

We examined for possible major complications of the procedure, including esophageal stricture, bleeding, perforation, and skin phototoxicity. Bleeding and perforation were not encountered in our cases. Esophageal stricture was the most frequent complication $(2 / 8,25 \%)$. In both the cases, multiple endoscopic balloon dilatations had to be performed. The incidence of esophageal stricture after TS-PDT is reported to be around $7.7-26.5 \%,{ }^{14,16}$ and in these reports, esophageal stricture was effectively treated by endoscopic balloon dilatation.
Skin phototoxicity, often associated with PS-PDT, was not observed in our study. It has been proven in a study conducted at our institution that TS-PDT outperforms PS-PDT in terms of safety, especially regarding skin phototoxicity. ${ }^{16}$

In this study, the L-CR rate was significantly high $(7 / 8,87.5 \%)$. Of these, T1 cases achieved $100 \%(6 / 6)$ L-CR, while T2 cases had 50\% L-CR (1/2). These results are similar to those of previous reports. Yano et al. have reported that the $\mathrm{L}-\mathrm{CR}$ rate of $\mathrm{T} 1$ failure lesions 
with TS-PDT was $100 \%(19 / 19)$, whereas that of T2 failure lesions was $57.1 \%(4 / 7) .{ }^{14}$ Surgical resection has been traditionally performed in ESCC patients with local failure after CRT. Salvage surgical resection is considered as an option for curative treatment for local failure after CRT in patients with ESCC. However, there are drawbacks in terms of invasiveness and safety. Salvage esophagectomy has a significantly higher incidence of anastomotic leaks (39\% vs. $7 \%$ ) and longer hospital stay ( 29 days vs. 18 days) compared to planned esophagectomy. ${ }^{17}$ Swisher et al. also reported that there was no significant increase in operative mortality compared to planned esophagectomy $(15 \%$ vs. $6 \%)$. It is important to recognize that this study was performed in a high-volume esophageal referral center with significant experience in high-risk esophagectomies. ${ }^{17}$ Perioperative mortality is likely to be higher in hospitals with low-volume esophageal procedures. ${ }^{18}$

Regarding ER, Hattori et al. reported that the overall survival rate of patients treated with salvage ER for locoregional failure after definitive CRT was $56 \%$ at 3 years. ${ }^{9}$ From a technical point of view, salvage ER is difficult and at risk of incompleteness if the residual tumor extends deeper than the submucosa. ${ }^{11}$

There are few reports on the long-term results of PDT. Amanuma et al. reported the 2-year OS rates were $79 \% .{ }^{16}$ In our study of a small number of patients, 2 -year OS rates was $87 \%$, which is a good result. In particular, all patients with $\mathrm{T} 1$ survived, suggesting that PDT may be an effective treatment in the long term. We consider TS-PDT to be superior to salvage surgical resection since it is less invasive and technically easier to perform than salvage ER. Furthermore, it needs to be highlighted that an appropriate follow-up schedule after CRT is necessary for the early detection of local failures.

This study has several limitations. It was a singlecenter retrospective study with a small sample size. However, we need to consider that the number of ESCC patients with local failure after CRT is limited to small cohorts, particularly those who underwent TS-PDT.

In conclusion, we opine that salvage TS-PDT is a safe and effective treatment for local failure after CRT in patients with ESCC. TS-PDT represents a promising treatment option, and we expect more patients with ESCC to benefit from TS-PDT hereon.

\section{REFERENCES}

1 Who's Certified [Internet]. Lyon: World Health Organization International Agency for Research on Cancer (IARC). GLOBOCAN 2018 [cited 2020 Dec 6]. Available from: https:// gco.iarc.fr/today/data/factsheets/cancers/6-Oesophagus-factsheet.pdf
2 Tachimori Y, Ozawa S, Numasaki H, Ishihara R, Matsubara H, Muro K, et al.; Registration Committee for Esophageal Cancer of the Japan Esophageal Society. Comprehensive registry of esophageal cancer in Japan, 2012. Esophagus. 2019;16:22145. DOI: 10.1007/s10388-019-00674-z, PMID: 31098822

3 Luo HS, Huang HC, Lin LX. Effect of modern high-dose versus standard-dose radiation in definitive concurrent chemoradiotherapy on outcome of esophageal squamous cell cancer: a meta-analysis. Radiat Oncol. 2019;14:178. DOI: 10.1186/ s13014-019-1386-X, PMID: 31623639

4 Fan CY, Su YF, Huang WY, Chao HL, Lin KT, Lin CS. Definitive radiotherapy dose escalation with chemotherapy for treating non-metastatic oesophageal cancer. Sci Rep. 2018;8:12877. DOI: 10.1038/s41598-018-31302-y, PMID: 30150679

5 Herskovic A, Martz K, Al-Sarraf M, Leichman L, Brindle J, Vaitkevicius V, et al. Combined chemotherapy and radiotherapy compared with radiotherapy alone in patients with cancer of the esophagus. N Engl J Med. 1992;326:1593-8. DOI: 10.1056/NEJM199206113262403, PMID: 1584260

6 Zhang Z, Liao Z, Jin J, Ajani J, Chang JY, Jeter M, et al. Dose-response relationship in locoregional control for patients with stage II-III esophageal cancer treated with concurrent chemotherapy and radiotherapy. Int J Radiat Oncol Biol Phys. 2005;61:656-64. DOI: 10.1016/j.ijrobp.2004.06.022, PMID: 15708243

7 Nishimura M, Daiko H, Yoshida J, Nagai K. Salvage esophagectomy following definitive chemoradiotherapy. Gen Thorac Cardiovasc Surg. 2007;55:461-5. DOI: 10.1007/s11748-0070157-z, PMID: 18049854

8 Miyata H, Yamasaki M, Takiguchi S, Nakajima K, Fujiwara Y, Nishida T, et al. Salvage esophagectomy after definitive chemoradiotherapy for thoracic esophageal cancer. J Surg Oncol. 2009;100:442-6. DOI: 10.1002/jso.21353, PMID: 19653262

9 Hattori S, Muto M, Ohtsu A, Boku N, Manabe T, Doi T, et al. EMR as salvage treatment for patients with locoregional failure of definitive chemoradiotherapy for esophageal cancer. Gastrointest Endosc. 2003;58:65-70. DOI: 10.1067/ mge.2003.306, PMID: 12838223

10 Makazu M, Kato K, Takisawa H, Yoshinaga S, Oda I, Saito Y, et al. Feasibility of endoscopic mucosal resection as salvage treatment for patients with local failure after definitive chemoradiotherapy for stage IB, II, and III esophageal squamous cell cancer. Dis Esophagus. 2014;27:42-9. DOI: 10.1111/ dote.12037, PMID: 23442160

11 Yano T, Muto M, Minashi K, Ohtsu A, Yoshida S. Photodynamic therapy as salvage treatment for local failures after definitive chemoradiotherapy for esophageal cancer. Gastrointest Endosc. 2005;62:31-6. DOI: 10.1016/S00165107(05)00545-6, PMID: 15990816

12 Yano T, Hatogai K, Morimoto H, Yoda Y, Kaneko K. Photodynamic therapy for esophageal cancer. Ann Transl Med. 2014;2:29. PMID: 25333005

13 Yano T, Muto M, Minashi K, Iwasaki J, Kojima T, Fuse N, et al. Photodynamic therapy as salvage treatment for local failure after chemoradiotherapy in patients with esophageal squamous cell carcinoma: A phase II study. Int J Cancer. 2012;131:1228-34. DOI: 10.1002/ijc.27320, PMID: 22024814 
14 Yano T, Kasai H, Horimatsu T, Yoshimura K, Teramukai S, Morita S, et al. A multicenter phase II study of salvage photodynamic therapy using talaporfin sodium (ME2906) and a diode laser (PNL6405EPG) for local failure after chemoradiotherapy or radiotherapy for esophageal cancer. Oncotarget. 2017;8:22135-44. DOI: 10.18632/oncotarget.14029, PMID: 28212527

15 National Cancer Institute (National Institutes of Health) [Internet]. Cancer Therapy Evaluation Program. Common terminology criteria for adverse events (CTCAE), version 4.0, DCTD, NCI, NIH, DHHS [updated June 14, 2010; cited Dec 8, 2020]. Available from: https://evs.nci.nih.gov/ftpl/CTCAE/ CTCAE_4.03/CTCAE_4.03_2010-06-14.xls
16 Amanuma Y, Horimatsu T, Ohashi S, Tamaoki M, Muto M. Association of local complete response with prognosis after salvage photodynamic therapy for esophageal squamous cell carcinoma. Dig Endosc. 2020;den.13730. DOI: 10.1111/ den.13730, PMID: 32434267

17 Swisher SG, Wynn P, Putnam JB, Mosheim MB, Correa AM, Komaki RR, et al. Salvage esophagectomy for recurrent tumors after definitive chemotherapy and radiotherapy. J Thorac Cardiovasc Surg. 2002;123:175-83. DOI: 10.1067/ mtc.2002.119070, PMID: 11782772

18 Swisher SG, DeFord L, Merriman KW, Walsh GL, Smythe R, Vaporicyan A, et al. Effect of operative volume on morbidity, mortality, and hospital use after esophagectomy for cancer. J Thorac Cardiovasc Surg. 2000;119:1126-34. DOI: 10.1067/ mtc.2000.105644, PMID: 10838528 